\title{
Modelling fast forms of visual neural plasticity using a modified second-order motion energy model
}

\author{
Andrea Pavan ${ }^{1, *}$, Adriano Contillo ${ }^{2,3}$, George Mather $^{4}$
}

1. Universität Regensburg, Institut für Psychologie, Universitätsstr. 31, 93053 Regensburg, Germany

2. Radboud University Nijmegen, Theoretical High Energy Physics Faculty of Science, 6500 GL Nijmegen, The Netherlands

3. Università degli Studi di Ferrara, Dipartimento di Fisica e Scienze della Terra, Via Saragat 1, 44122 Ferrara, Italy

4. University of Lincoln, School of Psychology, Brayford Pool, Lincoln LN6 7TS, UK

\section{*Corresponding author:}

Andrea Pavan

Universität Regensburg

Institut für Psychologie, Universitätsstr. 31, 93053

Regensburg, Germany

Tel: +49 (0)9419433582

Email: andrea.pavan@ur.de 


\begin{abstract}
The Adelson-Bergen motion energy sensor is well established as the leading model of low-level visual motion sensing in human vision. However, the standard model cannot predict adaptation effects in motion perception. A previous paper (Pavan et al. 2013) presented an extension to the model which uses a first-order RC gain-control circuit (leaky integrator) to implement adaptation effects which can span many seconds, and showed that the extended model's output is consistent with psychophysical data on the classic motion after-effect. Recent psychophysical research has reported adaptation over much shorter time periods, spanning just a few hundred milliseconds. The present paper further extends the sensor model to implement rapid adaptation, by adding a secondorder RC circuit which causes the sensor to require a finite amount of time to react to a sudden change in stimulation. The output of the new sensor accounts accurately for psychophysical data on rapid forms of facilitation (rapid visual motion priming, rVMP) and suppression (rapid motion after-effect, rMAE). Changes in natural scene content occur over multiple time scales, and multistage leaky integrators of the kind proposed here offer a computational scheme for modelling adaptation over multiple time scales.
\end{abstract}

Keywords: Short-term neural plasticity, Rapid visual motion priming, Rapid motion after-effect, Second-order RC integrator, Motion energy 


\section{Introduction}

The motion energy model (Adelson and Bergen 1985) is a biologically plausible model of low-level visual motion sensing. In its simplest form, it consists of four spatiotemporal filters oriented in space-time with pairs tuned to opposing directions, conventionally presented as two encoding rightwards motion and two encoding leftwards motion. The convolution of these four filters with a space-time representation of a moving stimulus (input image), produces four responses that are subsequently squared. Left $\left(E_{L}\right)$ and right $\left(E_{R}\right)$ motion energy are computed by summing the two left sensor outputs and the two right sensor outputs, respectively. The left-right motion direction of the input image is then computed by calculating opponent energy, i.e., the difference between the left and right motion energy (i.e., $E_{L}-E_{R}$ ).

Although the motion energy model in its basic form accounts for a range of psychophysical data, such as direction discrimination (Georgeson and Scott-Samuel 1999) and lateral masking (Rainville et al. 2002, 2005), it does not account for the effects of prolonged exposure to unidirectional motion (i.e., adaptation). After prolonged adaptation to directional motion, observation of either a stationary or a non-directional dynamic (i.e., counterphase flicker) pattern usually evokes an experience of motion in the opposite direction; the motion after-affect (MAE; see Mather et al. 2008 for a review). Recently we have extended the motion energy sensor by including an additional stage in the form of a RC automatic gain-control circuit operating in time domain (i.e., leaky integrator; Pavan et al. 2013). In particular, after squaring the output of the four space-time oriented filters, at each time instant the output of each convolution is multiplied by a factor that depends on the output of the sensor as a function of time in the recent past. This stage provides saturation of the motion sensors by reducing their sensitivity as a consequence of being exposed to a directional motion. We showed that the model predicts the exponential decay of the MAE as the adaptation-test interval increases (Hershenson 1993). Exponential decay is a key feature of leaky integrators (RC gain-control circuits), and has been reported as an electrophysiological and psychophysical property of the decay in motion adaptation (van de Grind et al. 2003; Taylor 1963; Vautin and Berkley 1977; Giaschi et al. 1993).

Generally, the MAE is induced by adapting for tens of seconds. However, there is plentiful physiological evidence that very brief stimuli presented in the RF of cells, or trains of electrical stimulation can induce both transient forms of suppression (i.e., adaptation) and facilitation (Priebeet al. 2002; Lisberger and Movshon 1999; Hempel et al. 2000; Nelson 1991; Chance et al. 1998; Finlayson and Cynader 1995; Stratford et al. 1996; Priebe and Lisberger 2002). When two 
stimuli are presented in rapid succession, the neural response to the latter stimulus is considerably reduced, a phenomenon well known as short-term synaptic depression (Nelson 1991; Finlayson and Cynader 1995; Varela et al. 1997, 1999; Chance et al. 1998; Lisberger and Movshon 1999; Priebe et al. 2002; Priebe and Lisberger 2002; Boudreau and Ferster 2005). Short-term synaptic depression has been reported within the striate cortex of cats (Boudreau and Ferster 2005) and also in area MT of monkeys (Lisberger and Movshon 1999; Priebe et al. 2002; Priebe and Lisberger 2002; Glasser et al. 2011). Priebe et al. (2002), for example, found that monkey MT neurons respond to a motion step with a transient-sustained firing rate. The transition from an initial high firing rate to a lower sustained rate occurs over a temporal window of $20-80 \mathrm{~ms}$ and can be considered as a form of shortterm adaptation. Accordingly, Glasser et al. (2011) found that in monkey MT a very brief exposure to directional motion $(67 \mathrm{~ms})$ produced direction-selective responses to subsequently presented stationary test stimuli. This is also compatible with a short-term form of motion adaptation.

On the other hand, other studies have reported that brief stimulation produces not only depression but also short-term facilitation for subsequently presented stimuli, which leads to an increase in neuronal responsiveness (Varela et al. 1997; Castro-Alamancos and Connors 1996; Hempel et al. 2000). Hempel et al. (2000) found strong short-term depression in neurons of the layer III of the rat prefrontal medial cortex during high-frequency electrical stimulation. However, they also found strong short-term facilitation during the early part of the electrical stimulation. In addition, short-term facilitation and depression occurred on a timescale ranging from tens to hundreds milliseconds. Hempel et al.'s (2000) study revealed that in many cortical circuits shortterm forms of depression and facilitation can coexist and compete (see also Varela et al. 1997 for similar results in the cat's striate cortex) and such synaptic dynamics are important in managing the shaping of neural responses and their interactions.

Recent psychophysical studies on humans indicate that such short-term forms of neural plasticity could provide the physiological substrate for rapid forms of motion after-effect (rMAE; suppression) and visual motion priming (rVMP; facilitation) (Pinkus and Pantle 1997; Kanai and Verstraten 2005; Pavan et al. 2009; Pavan et al. 2010; Pavan and Skujevskis 2013). Very brief exposure to directional stimuli can bias the perceived motion direction of a subsequently presented ambiguous test pattern (Kanai and Verstraten 2005; Pavan et al. 2009; Glasser et al. 2011; Pavan and Skujevskis 2013). In particular, depending on both the duration of the adaptation pattern and the duration of the blank inter-stimulus interval (ISI) between adaptation and testing, the perceived direction of an ambiguous test pattern can be biased towards the opposite direction (after-effect), or towards the same direction (priming) of the adaptation pattern (Pinkus and Pantle 1997). Using 
brief adaptation durations (e.g., 0.080 or $0.160 \mathrm{~s}$ ) and ISIs ( 0.040 or $0.120 \mathrm{~s}$ ) Kanai and Verstraten (2005) and Pavan et al. (2009) showed that the perceived direction of an ambiguous test pattern was biased towards the direction of the adaptation pattern, resulting in a rapid form of visual motion priming (rVMP). On the other hand, increasing the adaptation duration up to 0.320 or $0.640 \mathrm{~s}$, and using the same ISI levels induced a perceived bias in the opposite direction to the adaptation pattern (rMAE).

Rapid forms of adaptation thus constitute an important and distinctive property of neural processing in the visual system. The aim of the present study was to assess whether a further extension to the motion energy model can capture this characteristic. We tested the behaviour of the extended model against psychophysical data reported in Pavan et al. (2009) on rapid visual motion priming (rVMP) and rapid motion after-effect (rMAE), induced by brief exposures to directional moving patterns.

\section{Methods}

\subsection{Leaky integrators}

As in our earlier development of the motion energy model (Pavan et al. 2013), the new extension implements divisive feed-forward gain control in motion sensors using a 'leaky integrator' circuit, whose role is to simulate the saturation of the motion channel. A general feature of the leaky integrator is that the output signal at any time point is a fraction of the input, proportional to the magnitude of the input in the past.

The leaky integrator used in Pavan et al. (2013) consists of a parallel arrangement of a resistor $\left(R_{1}\right)$ and a condenser $\left(C_{1}\right)$, put in series to another resistor $\left(R_{2}\right)$ (Fig. 1A). For the sake of simplicity we call this circuit "first-order integrator". The output response (defined as the signal measured across $R_{l}$ ) to a step input is in turn a step that decreases exponentially with time, the typical temporal scale is: $\tau=R_{2} C_{1}$. More generally, the response $y(t)$ to a generic input signal $z(t)$ is equal to:

$$
y(t)=z(t)-\frac{e^{-(1+w) t / \tau}}{\tau} \int e^{(1+w) s / \tau} z(s) d s
$$

where $w=R_{2} / R_{1}$ is the parameter encoding the information about the asymptotic saturation level of the motion channel. The output of the first-order integrator in response to a step input is depicted in Fig. 2 . 
A

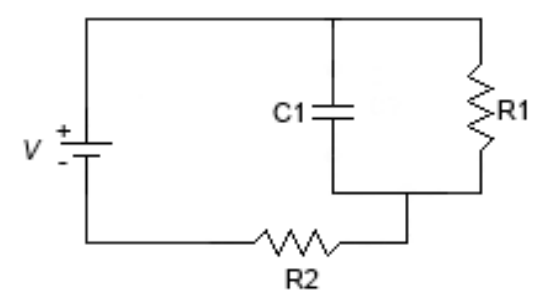

B

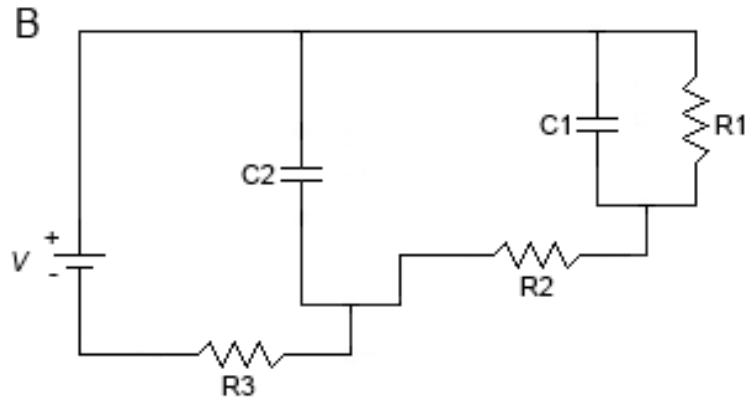

Fig. 1. Schematic representation of the first-order (A) and second-order (B) integrators. The voltage generators $V$ provide the input signal to the circuits, while the outputs are read across the resistors $R_{l}$. The embedding of the first-order integrator in the second-order integrator is clearly noticeable.

A second stage in the leaky integrator was added as follows: the whole first-order circuit was put in parallel with a second condenser $\left(C_{2}\right)$, and subsequently in series with a third resistor $\left(R_{3}\right)$, whose resistance is chosen to be much smaller than the others in order to avoid any alteration of the asymptotic response. We call this circuit "second-order integrator" (Fig. 1B). The output of the second-order integrator is given by the following function:

$$
y(t)=e^{-t / T} \frac{w-\tau / T}{T(1+w)-\tau} \int e^{s / T} z(s) d s+e^{-(1+w) s / \tau} \frac{1}{T(1+w)-\tau} \int e^{(1+w) s / \tau} z(s) d s
$$

featuring the new time constant $T=R_{3} C_{2}$.

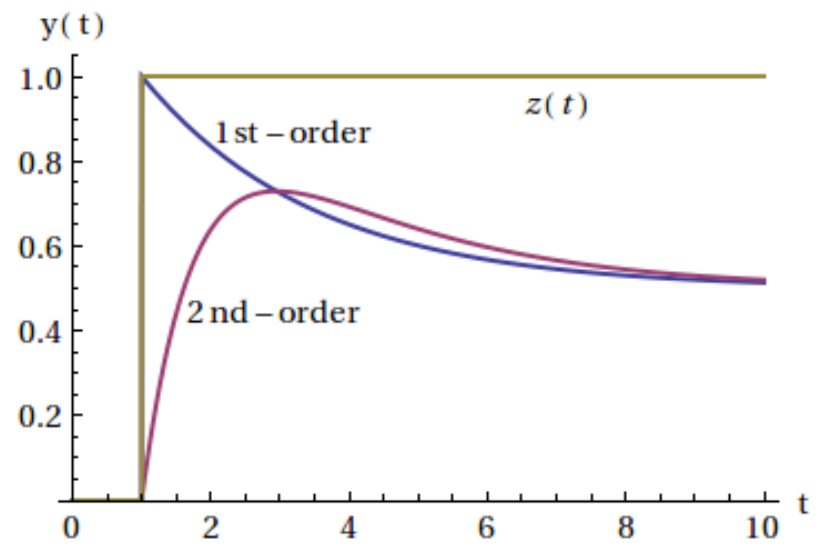

Fig. 2. Response of the first- (blue) and second-order (purple) integrators to a step input signal (gold line). Here the asymptotic response $y=z / 2$ was obtained setting $w=1$, while the time constants were $\tau=5$ and $T=0.8$.

The second-order circuit is fully described by the parameters $\{w, \tau, T\}$, where $w$ regulates the asymptotic magnitude of the response, $\tau$ refers to the timescale needed to reach the asymptote and $T$ the time needed to reach the maximum response when the input is switched on. As mentioned 
earlier, $T$ should be much smaller than $\tau$ in order to keep the two effects (i.e., switching on and asymptotic decreasing) separate. The response of the second-order circuit to a step input is shown in Fig. 2. The comparison between the two responses makes clear the difference between the first- and second-order integrator. In particular, the second-order circuit takes a finite amount of time to reach maximum response, but then it accurately mimics the first-order integrator in its exponential decay.

Fig. 3 shows the theoretical output of the first- and second-order integrators. Consider, for example, two motion channels, tuned to left and right respectively, stimulated with a leftward moving adapting pattern with duration $t_{A}$, followed by a blank interval with duration $t_{I S I}$ and then a test pattern that stimulates both channels equally. The difference between the outputs of the two sensors is $\Delta y(\mathrm{t})=y_{\mathrm{L}}(\mathrm{t})-y_{\mathrm{R}}(\mathrm{t})$. A negative difference (i.e., suppression) indicates rMAE, whereas a positive difference (i.e., facilitation) indicates rVPM. Fig. 3A shows the two input signals $z_{\mathrm{L}}$ and $z_{\mathrm{R}}$, having chosen $t_{A}=1$ and $t_{I S I}=1$, and the output difference as functions of time. It can be clearly seen that the output at the end of the blank interval indicates rVPM. Fig. 3B shows a similar situation, this time with $t_{I S I}=2$. Increasing the blank interval clearly reduces the magnitude of the priming that is now almost zero. Fig. $3 \mathrm{C}$ and $\mathrm{D}$ represent the equivalent situation, this time having set $t_{A}=4$. The output at the end of the blank interval in panel $\mathrm{C}$ is similar to the one in panel $\mathrm{A}$, while the one in panel $\mathrm{D}$ is already well inside the region of rMAE. The lesson we draw is that elongating the adaptation results in a reduced priming duration. In the next section the second-order integrator is embedded in the motion energy model. 

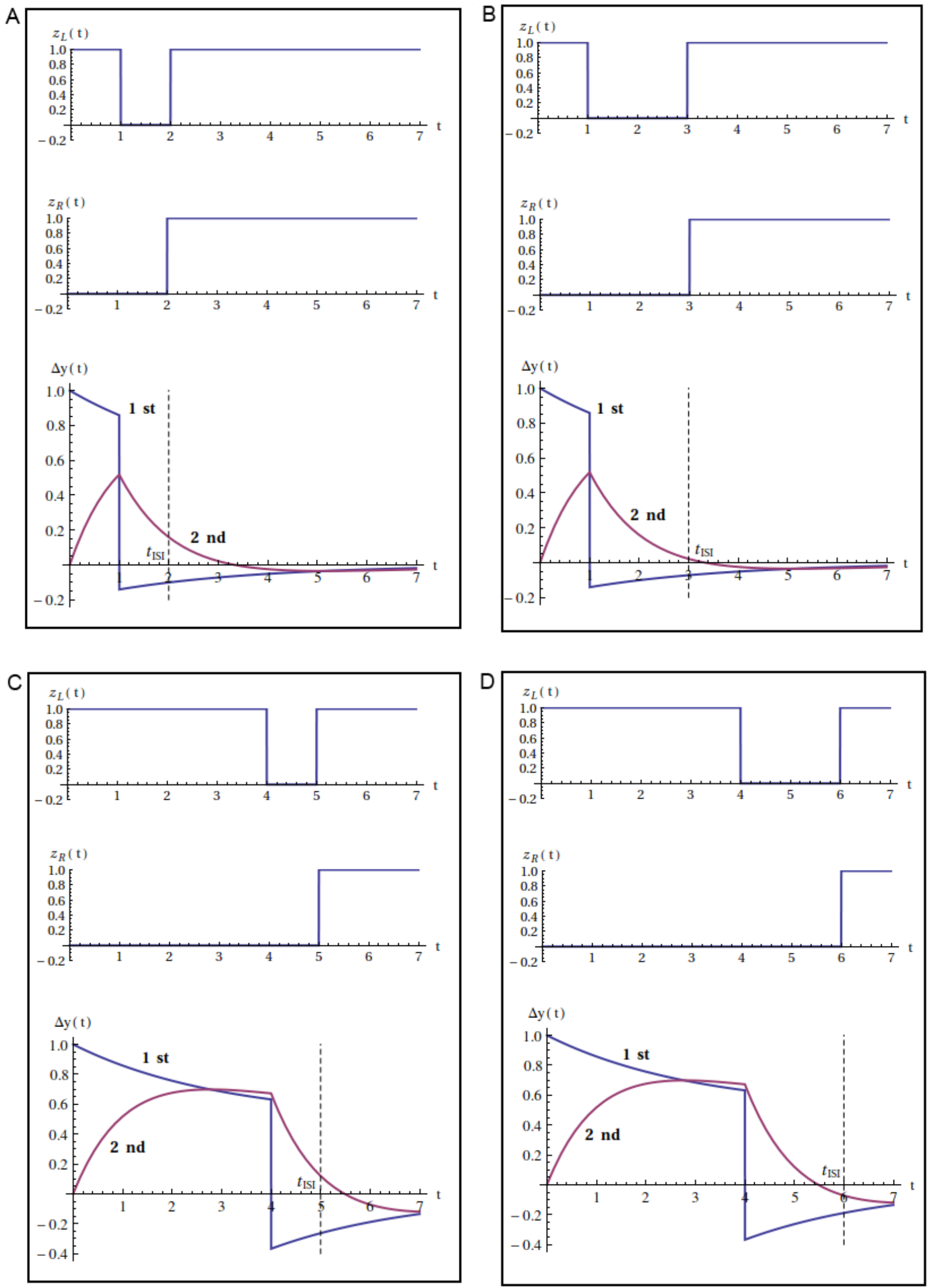
Fig. 3. Time dependence of left (top sub-panels) and right (mid sub-panels) input stimuli and of the left-right output difference for a fist- (blue) and second-order (purple) integrator. Adaptations grow from top $\left(t_{A}=1\right.$, panels $\mathrm{A}$ and $\left.\mathrm{B}\right)$ to bottom $\left(t_{A}=4\right.$, panels $\mathrm{C}$ and $\left.\mathrm{D}\right)$, while the adapting-target blank intervals (ISIs) grow from left ( $t_{I S I}=1$, panels A and C) to right ( $t_{I S I}=2$, panels B and D). The dashed lines indicate the beginning of the test stimulus. Here it was set $w=1, \tau=6$ and $T=1.2$ respectively.

\subsection{Input Stimuli}

The input stimuli for the model were based closely on those used by Pavan et al. (2009), and consisted of a spatiotemporal representation of a leftward drifting sinewave grating (adapting pattern), ISI and a counterphase flickering grating (test) (Fig. 4). The input stimuli were encoded in space-time (i.e., $x t$ ) with the spatial dimension covering $8 \mathrm{deg}$ sampled at intervals of $0.05 \mathrm{deg}$, and the temporal dimension of 4-s sampled at intervals of $0.005 \mathrm{~s}$ (much shorter that used in the psychophysical stimulus, in order to obtain a smoother spatiotemporal representation of the model stimulus; however frame-to-frame phase-shifts created the same drift velocity for both psychophysical and model stimuli) (see section 3.1 for further details on psychophysical stimuli). Adapting stimuli spanned 16, 32, 64 or 128 frames, for $0.08,0.160,0.320$ and $0.640 \mathrm{~s}$ adaptation duration respectively, against a grey background. ISI durations were: 8, 24, 96, 200 and 400 frames, corresponding to $0.040,0.120,0.480,1$ and $2 \mathrm{~s}$.

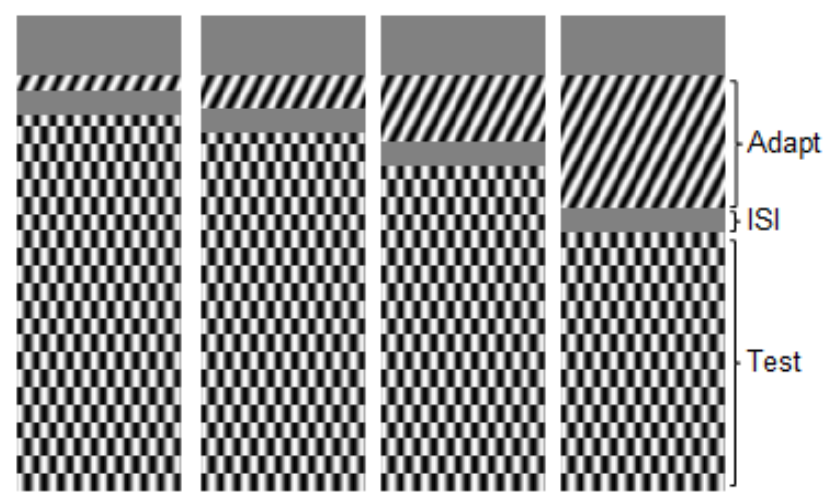

Fig. 4. Representation of a subsample of the input stimuli. The input stimuli consisted of a spacetime $(x t)$ representation of an adapting grating drifting leftward and with duration of $0.08,0.160$, 0.320 and $0.640 \mathrm{~s}$ (panels from left to right). Adapting stimulus was followed by an inter-stimulus interval (ISI: $0.120 \mathrm{~s}$ in the images) and a directionally ambiguous (i.e., counterphase-flicker) test pattern. 


\subsection{Motion Energy Model}

In order to better understand the second-order Motion Energy Model we briefly introduce both the standard Motion Energy Model (Adelson and Bergen, 1985) and its modification through the leaky integrator stage. The spatial and temporal profiles of the filters of the model covered 2.25 deg of space and 1-s of time. Spatial filter profiles were even $(E V)$ and odd $(O D)$ Gabor functions of the form:

$$
\begin{aligned}
& E V(x)=\cos (2 \pi f x) e^{-(x / \sigma)^{2}} \\
& O D(x)=\sin (2 \pi f x) e^{-(x / \sigma)^{2}}
\end{aligned}
$$

where $f$ is $1.1 \mathrm{cpd}$ and $\sigma$ is $0.5 \mathrm{deg}$. Temporal filters had the following form, taken from Adelson and Bergen (1985) Eq. (6):

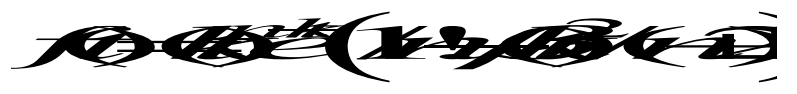

The value of $k$ scales the response into time units and was set to 100, while $n$ sets the vertical (temporal) width of the filter (Challinor and Mather 2010). The parameter $n$ was equal to 9 for the slow temporal filter and 6 for the fast temporal filter, as used in previous modelling (Emerson et al. 1992; Strout et al. 1994; Takeuchi et al. 1997; Bergen and Wilson 1985; Rushton 1962). The parameter $\beta$ reflects the weighting of the negative phase of the temporal impulse response relative to the first positive phase and was set to 0.9 (Strout et al. 1994; Takeuchi and De Valois 1997; Fuortes and Hodgkin 1964). The product of the even and odd spatial profiles [i.e., $E V(x)$ and $O D(x)]$ with the two temporal profiles $\left[f_{\text {slow }}(t)\right.$ and $\left.f_{\text {fast }}(t)\right]$ creates four (space-time) separable filters (first layer of the model; Figure 2). These filters were combined to obtain in turn four sensors oriented in space-time; two oriented for leftward motion and two for rightward motion (second layer of the model; Figure 2). The two members of each pair are approximately 90 deg out of phase with each other (Adelson and Bergen 1985). Convolving these four filters with the same input image gives four response matrices that are subsequently squared (first and second layers of the model; Fig. 5). We label the matrices resulting from this squaring as $R_{L 1}, R_{L 2}, R_{R 1}$, and $R_{R 2}$.

The modified Motion Energy Model was obtained by implementing an adapting stage by introducing the modified $R C$ integrator (third layer of the model; Fig. 5). That is, we first averaged the output $z(t)$ of the convolutions over the whole spatial range and over the sampling interval $\Delta t=$ $0.005 \mathrm{~s}$. We can schematically write this averaging as

$$
\langle z\rangle(t)=\frac{1}{X} \int_{X} z(t, x) d x
$$


where $X$ is the spatial size of the output matrix. Notice that the $\langle z\rangle(t)$ will now act as the input of the integrator stage. Then at each time slice (row in the output matrix), the output of each convolution stage is multiplied by the factor

$$
r(t)=\frac{y(t)}{\langle z\rangle(t)}=1-\frac{e^{-(1+w) t / \tau}}{\langle z\rangle(t) \tau} \int_{0}^{t} e^{(1+w) s / \tau}\langle z\rangle(s) d s
$$

in the case of the first-order integrator or by the factor

$$
r(t)=\frac{1}{\langle z\rangle(t)}\left(e^{-t / T} \frac{w-\tau / T}{T(1+w)-\tau} \int e^{s / T}\langle z\rangle(s) d s+\frac{e^{-(1+w) s / \tau}}{T(1+w)-\tau} \int e^{(1+w) s / \tau}\langle z\rangle(s) d s\right) \quad \text { Eq.8 }
$$

with the second-order integrator. For example, $r_{L 1}(t)$ will be obtained taking as $\langle z\rangle(t)$ the spatial average of $R_{L 1}(x, t)$. Notice that the above formulas directly derive from Eq. 1 and 2. Formally this can be written as follows:

$R_{L 1}^{\prime}(x, t)=R_{L 1}(x, t) r_{L 1}(t)$

$R_{L 2}^{\prime}(x, t)=R_{L 2}(x, t) r_{L 2}(t)$

$R_{R 1}^{\prime}(x, t)=R_{R 1}(x, t) r_{R 1}(t)$

$R_{R 2}^{\prime}(x, t)=R_{R 2}(x, t) r_{R 2}(t)$

Then, as required in the standard model, we summed the responses derived from the two pairs of filters to compute leftward and rightward motion energies. The output matrices are respectively defined as:

$E_{L}(x, t)=R_{L 1}^{\prime}(x, t)+R_{L 2}^{\prime}(x, t)$

$E_{R}(x, t)=R_{R 1}^{\prime}(x, t)+R_{R 2}^{\prime}(x, t)$

Opponent energy is then computed using the following measure of net Energy:

$$
E_{n t}=\frac{E_{L}-F_{R}}{E_{\text {llk }}}
$$

with a normalization factor, called flicker energy (Georgeson and Scott-Samuel 1999), defined as an average over the whole output matrix:

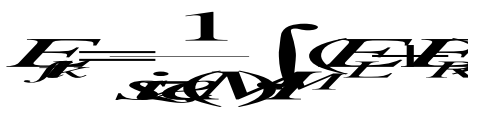

where $\operatorname{size}(M)$ schematically indicates the size of the output matrix. 
All the quantities described so far can be computed for both the first- and the second-order integrators. Since the first-order integrator was described in detail in Pavan et al. (2013), we now focus on the second-order integrator.

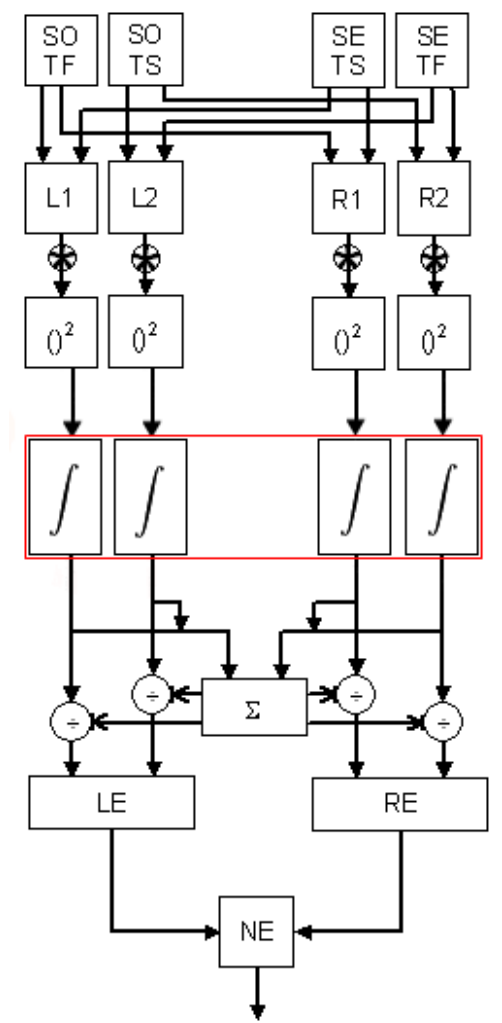

Fig. 5. The extended motion energy model. The red frame highlights the integrator stage that is located after the squaring stage (see text for more details).

Because of its definition net Energy depends on the choice of the parameters $\{w, \tau, T\}$. Thus, it is important to select the values that best fit the behavioural data reported in Pavan et al. (2009). Let us start by extracting from the net Energy the numerical predictions to be tested against the behavioural data that are presented in the form of a table spanning a set of adaptation durations and ISI levels. We selected the first combination of $t_{A}$ and $t_{I S I}$ and feed the extended Motion Energy Model with the corresponding input pattern, obtaining a first net Energy matrix. We subsequently averaged the matrix along the spatial dimension, obtaining a vector that encodes the mean net Energy as a function of time

$$
\bar{\varepsilon}(t)=\frac{1}{X} \int_{X} E_{n e t}(t, x) d x
$$


The normalized output was then defined as minus the ratio between the mean net Energy evaluated at the end of the inter-stimulus interval (i.e., at the onset of the test stimulus) and the minimum value of the first-order mean net Energy, defined in the same way

$$
p(t)=-\frac{\bar{\varepsilon}\left(t_{A}+t_{I S I}\right)}{\bar{\varepsilon}_{1 s t}\left(t_{A}\right)}
$$

This choice of the normalization factor was made on the basis of Pavan et al. (2013) as the present generalization is expected to recover exactly the same scheme if $T$ is neglected. In Pavan et al. (2013) the denominator of Eq. 14 represented a lower bound for the mean net Energy, as it described the amount of rMAE experienced right at the end of the adaptation period by an ideal observer lacking in the second stage of the leaky integrator. It is therefore natural to extend this role to the present case, since the addition of the second stage causes the output values in that regime to rise. Notice that Eq. 14 is always contained in the interval $[-1,1]$, indicating rMAE for negative values and rVMP for positive values. Therefore, its magnitude can be interpreted as a measure of the strength of the perceived motion and can be compared to the first entry of the psychophysical data. The procedure is then iterated for each combination of $t_{A}$ and $t_{I S I}$ and the results are compared with the data, computing the root mean square error (RSME) of each combination and summing them together to have a measure of the quality of the modelling. We call such measure total Root Mean Square Error (TRMSE).

In the supplementary material we provide the MATLAB code of the second-order Motion Energy Model.

\section{Results}

\subsection{Psychophysical data}

The output of the extended motion energy model was fitted to a subset of the data reported in Pavan et al. (2009). Eight observers participated in the Experiment. They sat in a dark room 57 $\mathrm{cm}$ from the screen. Viewing was binocular. They were instructed to fixate a point at the center of the screen. All subjects had normal or corrected-to-normal visual acuity.

Stimuli were vertically oriented Gabor patches (full width of 8 deg at half maximum amplitude and a spatial frequency of $1 \mathrm{c} / \mathrm{deg}$ ). Adapting stimuli drifted either leftward or rightward; test patterns were counterphase flickered Gabor patches, as in the model stimulus. Directional and ambiguous stimuli were obtained by shifting the phase of the sinusoidal carrier. For example, a horizontal directional stimulus was created by shifting the phase left or right ( $\pm 90 \mathrm{deg})$. This phase 
shift was chosen because Pinkus and Pantle (1997) showed that visual motion priming is maximum at a 90 deg phase shift. Ambiguous test patterns were created by shifting the phase 180 deg every $0.080 \mathrm{~s}$. Velocity of the test stimulus was equal to that of the adapting stimulus $(6.25 \mathrm{deg} / \mathrm{s})$. The motion direction of the adapting stimulus was balanced and randomized across trials.

Four adaptation durations were used: $0.080,0.160,0.320$, and 0.640 s. After a variable ISI $(0.040,0.120,0.480,1,2$ and $5 \mathrm{~s})$ during which the display was blank (except the fixation point, that was present also during the ISI), an ambiguous test pattern was presented for $0.320 \mathrm{~s}$.

Observers judged whether the test stimulus was moving in the same direction or opposite to the adaptation pattern. There were a total of 48 conditions; 2 adapting directions $\mathrm{x} 4$ adapting durations x 6 adapting-test intervals (ISIs). Twenty trials were performed for each condition, and the order of conditions was randomized across trials.

In the present study we consider only ISIs ranging from 0.040 to $2 \mathrm{~s}$, because these ISIs produced reliable rVMP and rMAE at the adapting durations used (i.e., 0.080, 0.160, 0.320 and 0.640 s; see Kanai and Verstraten 2005 and Pavan et al. 2009).

For the purposes of comparison with model output we reanalysed a subset of the Pavan et al.'s (2009) psychophysical data to assess whether a particular combination of adaptation duration and ISI induced rVMP or rMAE above chance. To achieve more statistical power the data from the two adapting directions were pooled.

We performed a series of Bonferroni-Holm corrected two-sided one-sample t-tests (Holm 1979; Groppe et al. 2011), separately for each adaptation duration, and across all the ISI levels. The result showed that using adaptation lasting $0.080 \mathrm{~s}$ with an ISI of $0.040 \mathrm{~s}$ the perceived direction of the ambiguous test pattern was significantly biased towards the direction of the adaptation stimulus ( $84 \%$ of response in the same direction) $t_{7}=11.26, p=0.0001$, resulting in a rVMP. The t-tests did not report any other significant rVMP effects. For longer adaptation durations the perceived direction of the ambiguous test pattern was biased towards the direction opposite to the adapting stimulus (rMAE). In particular, adapting for $0.320 \mathrm{~s}$ biased the perceived direction of the test stimulus opposite to the direction of the adaptation pattern after $0.120 \mathrm{~s}$ (17\% of response in the same direction) $t_{7}=-5.99, p=0.005$ and $1 \mathrm{~s}\left(36 \%\right.$ of response in the same direction) of ISI $t_{7}=-4.66$, $\mathrm{p}=0.008$. Adapting for $0.640 \mathrm{~s}$ produced a significant bias towards the opposite direction of the adapting stimulus only after $0.120 \mathrm{~s}$ of ISI $(15 \%) t_{7}=-7.13, p=0.0001$. 


\subsection{The Best Fitting Procedure}

To extract the best fitting values for the second-order integrator parameters $\{w, \tau, T\}$ we started with guessing $w=1, \tau=0.5 \mathrm{~s}$ and $T=0.05 \mathrm{~s}$ for which we obtained a TRMSE $(1,0.5 \mathrm{~s}, 0.05 \mathrm{~s})$ $=0.282$. Subsequently we used a Metropolis-Hastings algorithm, consisting in assigning a random transition proposal (from a uniformly distributed randomization) and evaluating the TRMSE in the new position. In other words, we randomly generated a (small) modification of the parameters and compared the new TRMSE value to the previous one: the smaller the new value was with respect to the previous one, the higher was the probability to accept the new position as the (temporary) best fitting choice. In particular, the acceptance probability is defined as the ratio between the former and the latter TRMSE.

Iterating this procedure for several thousand steps $(\sim 15 \mathrm{~K})$, the best fitting choice spans the three-dimensional space of parameters and rapidly falls (<1000 steps) into an almost-degenerate curve of minima, as it can be seen in Fig. 6. It drifts along this curve for a few thousand steps, then ( 4000 steps) starts oscillating around some value: $w \sim 10, \tau \sim 3 \mathrm{~s}$ and $T \sim 0.04 \mathrm{~s}$. The transition from the drifting to the oscillating phase is clearly visible in Fig. 6. All the positions selected from here are almost equivalent, so we select the one that gives the smaller TRMSE $(11.779,3.704 \mathrm{~s}$, $0.036 \mathrm{~s})=0.131$.
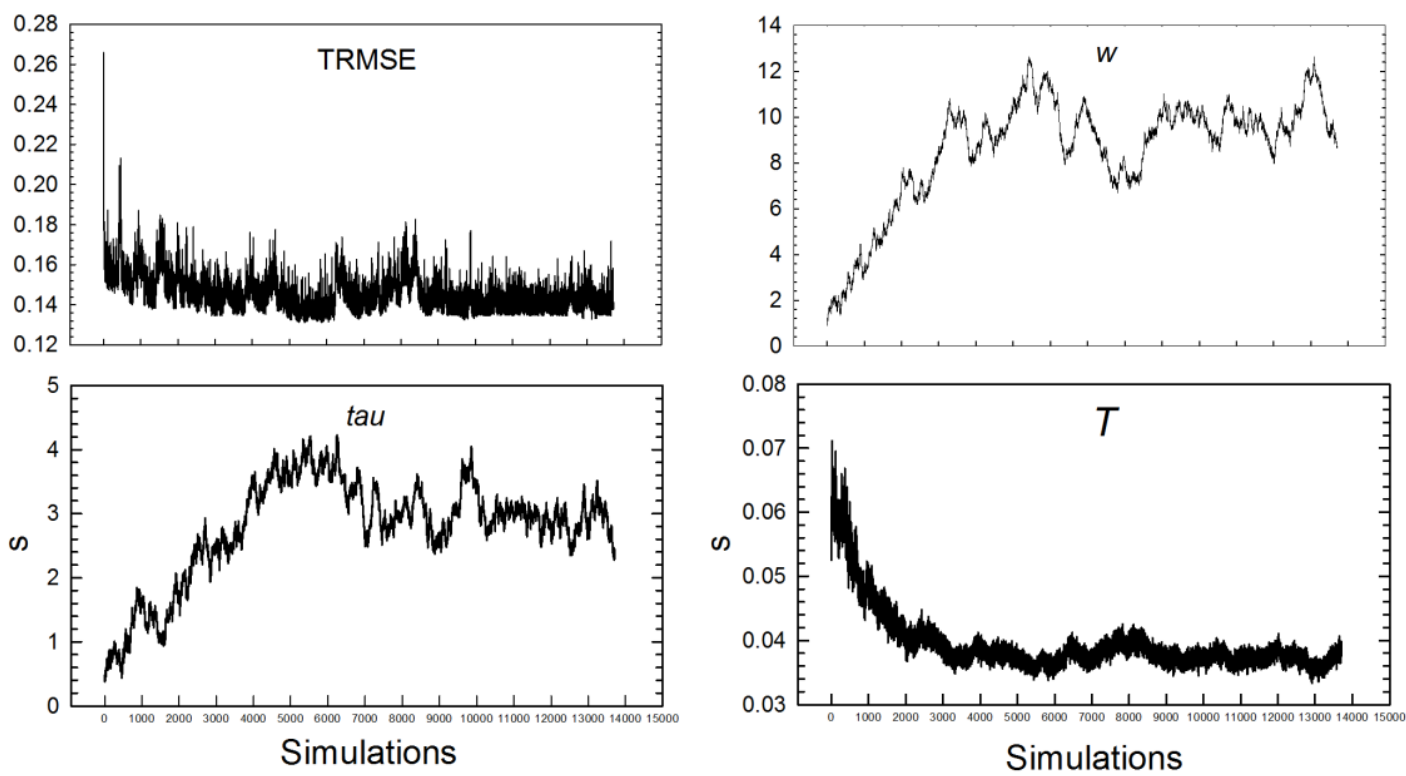

Fig. 6. Output of the Metropolis-Hastings algorithm. The nearly flat distribution of TRMSE values indicates the presence of the almost-degenerate curve of minima. The drifting-to-oscillating transition is clearly noticeable in the distributions of $w, \tau$ and $T$. 
The extended motion energy model incorporating the second-order integrator accurately fits the behavioural data on rapid forms of motion after-effect (rMAE) and visual motion priming (rVMP) (Fig. 7).
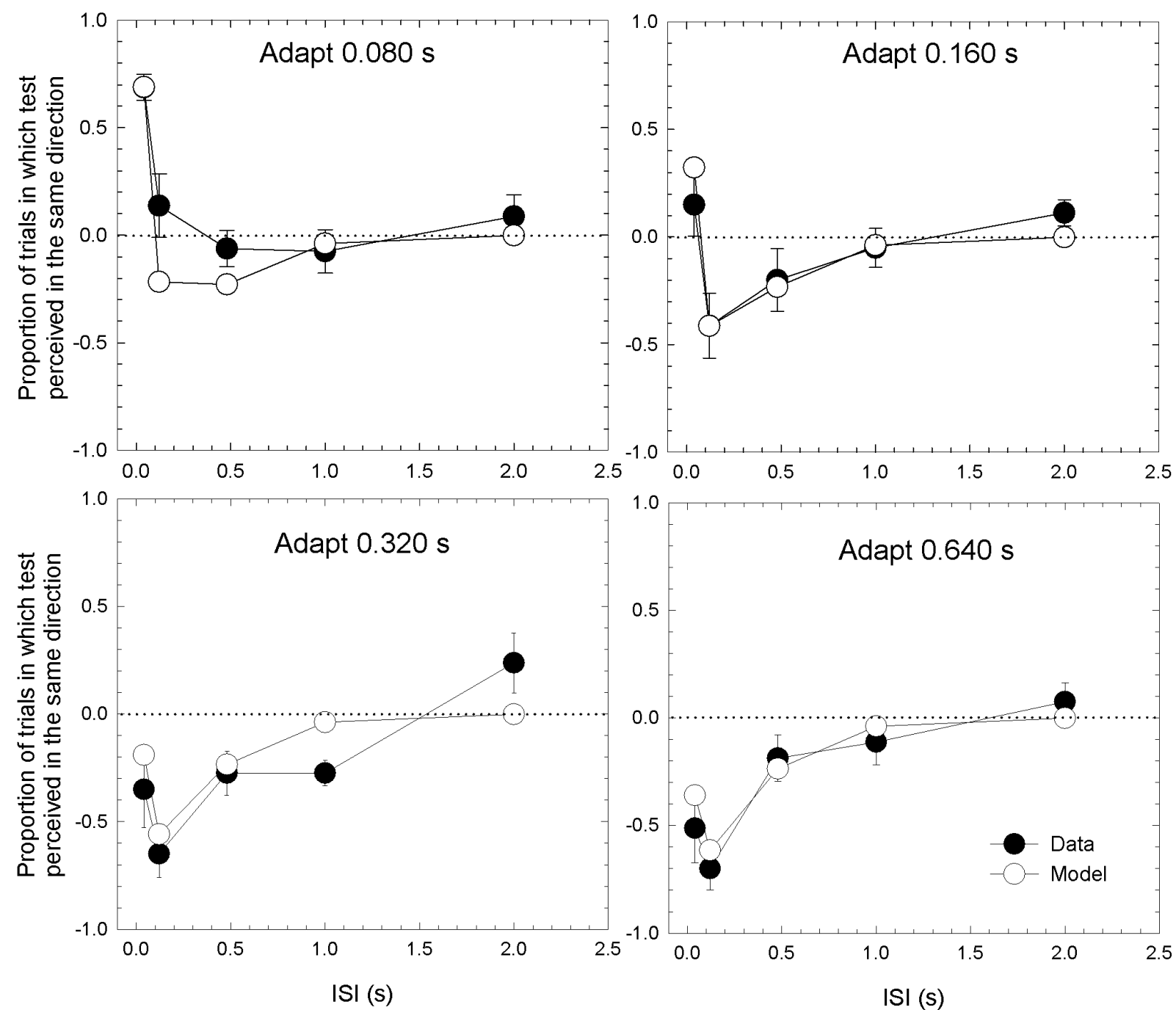

Fig. 7. Extended motion energy model output fitted to the psychophysical data. The proportion of trials in which observers judged the test stimulus as drifting in the same direction as the adaptation stimulus is shown as a function of the ISI (s). Panels show the results for adaptation durations of $0.080 \mathrm{~s}, 0.160 \mathrm{~s}, 0.320 \mathrm{~s}$, and $0.640 \mathrm{~s}$ (filled circles). Positive values on the ordinate indicate rVMP whereas negative values indicate rMAE. The dashed line is the level at which there was no perceived rMAE or rVMP. Empty circles indicate the model's output. Error bars \pm SE. 


\section{Discussion}

With the addition of a second RC-stage to the leaky integrator, the motion sensor requires a finite amount of time to fully react to a sudden change in stimulation. As a result, after very brief adaptation (e.g., $0.080 \mathrm{~s}$ ), the motion sensor with the highest response (i.e., the lowest saturation) at the onset of the test pattern signals motion in the same direction as the adapting pattern (i.e., rVMP). On the other hand, following longer exposure to directional motion the sensor signals a direction opposite to the adapting pattern (i.e., rMAE). Thus, the final output of the second-order model shows effects which mirror those reported by previous psychophysical studies on fast forms of motion adaptation (Kanai and Verstraten 2005; Pavan et al. 2009, 2010; Glasser et al. 2011; Pavan and Skujevskis 2013). The extended Motion Energy Model does not account for another effect previously reported by Kanai and Verstraten (2005) and Pavan et al. (2009, 2010), called "Perceptual Sensitization" (PS). PS is a later facilitation that appears following longer adaptation durations (e.g., 320 and $640 \mathrm{~ms}$ ) and after ISIs > $2 \mathrm{~s}$, for which the ambiguous test pattern is biased towards the same direction to that of the adapter. Hempel et al. (2000) also reported the presence of a slower form of enhancement of synaptic transmission occurring on the timescale of seconds to tens of seconds in addition to short-term facilitation and suppression, in the layer $\mathrm{V}$ of the rat prefrontal medial cortex. The authors found that the decay time of such enhancement was best fitted by a sum of two exponentials with a fast decay time of $7 \mathrm{~s}$ and a slow decay time of $71 \mathrm{~s}$. Such enhancements are classically labelled as "augmentation" and "posttetanic potentiation" for the short and long phases, respectively (Zengel and Magleby 1982). So far, physiological data support the notion that augmentation is seen more clearly in associative areas (Hempel et al. 2000).

In a previous study (Pavan et al. 2010) we pointed out some similarities between augmentation and PS in terms of decay time. However, the fact that augmentation has been observed mainly at high-level areas suggests that PS might be confined to higher level of motion processing and thus being more susceptible to attentional and expectation influences (Seidemann and Newsome 1999; Treue and Maunsell 1996, 1999; Rees et al. 1997; Haug et al. 1998; Buchel et al. 1998; Huk et al. 2001). Indeed, Daelli et al. (2010) reported a similar effect using complex objects as stimuli. In particular, when adapting to complex objects and testing with morphs (ambiguous stimuli), they found a switch from adaptation to a priming effect as the temporal delay between a prototype and an ambiguous test stimulus was increased (i.e., up to $3 \mathrm{~s}$ ). The authors argued that complex interactions between networks, including expectations for image disambiguation, are likely to be mediated by synaptic back-projections to early visual cortices (Rolls 1989). Since the neural mechanisms underlying PS and its interaction with attentional 
processes and expectations are still not clear, in the present study we focused on the early components of neural dynamics (i.e., facilitation and suppression) (Nelson 1991; Finlayson and Cynader 1995; Castro-Alamancos and Connors 1996; Varela et al. 1997, 1999; Chance et al. 1998; Lisberger and Movshon 1999; Hempel et al. 2000; Priebe et al. 2002; Priebe and Lisberger 2002; Boudreau and Ferster 2005; Glasser et al. 2011). Additionally, there is psychophysical evidence that PS appears following brief adaptation to ambiguous complex stimuli (Daelli et al. 2010) and to directionally ambiguous patterns (Kanai and Verstraten, 2005; Pavan et al. 2010), for which a motion energy detector cannot produce any response.

Hempel et al. (2000) suggested that brief periods of synaptic activity may be able to transiently shift a set of interconnected cortical neurons into a state in which recurrent excitation is sufficiently strong to support persistent activity. One intriguing possibility is that such neural dynamics could potentially provide the neural mechanism for attractor networks, considered to play an important role in a variety of visual and high-level cognitive functions (e.g., visual short-term memory, working memory, associative memory, spatial orientation; Amit 1989; Daelli and Treves 2010). For example, Daelli and Treves (2010) reported that an attractor network model can account for short-term priming and adaptation effects. In an attractor network model the strength of the associations between attractors can determine a transition between the activity elicited by the prime and the neural response to the target. On the other hand, an attractor network model can account for the switch between priming and aftereffects if it is endowed with firing rate adaptation that pushes the network away from its current attractor.

In experimental protocols involving brief adaptation periods, the first stage of the integrator, with time constant of some seconds, produces exponential decay that is characteristic of the rMAE. The second stage, with a time constant of tens of milliseconds, produces the non-instantaneous response onset that is responsible for rVPM. Using protocols to measure the classical MAE (longer adaptation periods), the first stage alone is sufficient to produce an adequate match to psychophysical data but the underlying motion processes may well possess a second stage integrator as well. Given the psychophysical evidence for multiple adaptation sites in the MAE, the different sites may possess integrators with different time constants, which mediate psychophysical effects at different adaptation durations. However, a weakness of the model is that it is descriptive in the sense that it cannot make predictions regarding the presence of specific impedances in particular cortical areas of the human brain. The results of our analysis could be viewed as description of part of a vast population of parasite impedances, most likely hidden in the transmission lines that carry the electric signals through the cortical areas involved in motion 
processing (e.g., V1/V2, V3+ and MT). Thus, no exact prediction is made about the location and distribution of such impedances. The modified Motion Energy Models (both first- and secondorder) can be further developed, for example, by introducing spatiotemporal filters selective to a range of contrast levels, spatial and temporal frequencies and speeds in order to account for spatiotemporal dynamics of the perceptual outcomes related to rapid and prolonged motion adaptation. This would produce a set of models useful to make specific predictions on future experiments on motion processing and would allow inferences about the cortical sites in which parasite impedances are likely to be implemented. This is because the selectivity to spatial contrast, temporal frequency and speed is implemented in specific cortical areas involved in motion processing (e.g., V1, V2, V3, V3A, V3B, V4v and MT/MST; Lingnau et al. 2009). It may also be advantageous to incorporate top-down influences, such as an attentional mechanism, into the design. This would account for a number of attention-related effects as adaptation to ambiguous motion and perceptual sensitization. There are indeed plenty of examples in literature that show how attention can clearly bias the percept of directionally ambiguous moving stimuli like the attention-based motion (Treue and Maunsell 1996, 1999; Cavanagh 1992; Verstraten and Ashida 2005), or induce the MAE (Culham et al. 2000).

There have been a number of reports of apparent asynchrony between changes of different visual attributes, such as between motion and colour (Moutoussis and Zeki 1997; Arnold and Clifford 2001): When a stimulus oscillates in both colour and motion, changes in colour appear to be asynchronous with changes in motion, by $50-100 \mathrm{msec}$. The apparent asynchrony may be due to differences in the relevant time constants of the leaky integrators serving different neural subsystems. The time constants determine the time required for each sub-system to react to a change in stimulation, so if they are different the responses of the sub-systems will change at slightly different times. One potential area for future investigation would be to apply RC-gain control circuits to models which encode other stimulus attributes, such as position, and test the hypothesis that perceptual asynchrony is due to differences in the time constants of the leaky integrators. A second area of investigation would be to extend the model into two spatial dimensions and test whether it can predict adaptation-induced changes in apparent direction.

According to Wark et al. (2009) the dynamics of adaptation should reflect a balance between adapting rapidly to avoid short-term saturation, and adapting slowly to avoid instability in the absence of changes in image statistics. Changes in natural image content occur over multiple time scales, so adaptation in the visual system might be expected to occur over a correspondingly diverse range of time scales. Multi-stage integrators of the kind used here successfully to model rapid and 
slow forms of motion adaptation offer a computational scheme for modelling adaptation over multiple time scales.

\section{Acknowledgements}

Author AP was supported by grants from the Alexander von Humboldt Foundation, Author AC was supported by the Deutsche Forschungsgemeinschaft (DFG) within the Emmy-Noether program (Grant SA/1975 1-1) and by the Università degli Studi di Ferrara, Author GM was supported by the University of Lincoln.

\section{References}

Adelson, E. H., \& Bergen, J. R. (1985). Spatiotemporal energy models for the perception of motion. Journal of the Optical Society of America A, 2, 284-299.

Amit, D. J. (1989). Modeling brain function. Cambridge University Press, New York.

Arnold, D. H., \& Clifford, C. W. (2002). Determinants of asynchronous processing in vision. Proceedings of the Royal Society of London, B269, 579-583.

Bergen, J. R., \& Wilson, H. R. (1985) Prediction of flicker sensitivities from temporal three pulse data. Vision Reseach, 25, 577-582.

Boudreau, C. E., \& Ferster, D. (2005). Short-term depression in thalamocortical synapses of cat primary visual cortex. Journal of Neuroscience, 25, 7179-7190.

Buchel C, Josephs O, Rees G, Turner R, Frith CD, Friston KJ (1998) The functional anatomy of attention to visual motion. A functional MRI study. Brain 121: 1281-1294.

Castro-Alamancos, M., \& Connors, B. W. (1996). Short-term synaptic enhancement and long-term potentiation in neocortex. Proceedings of the National Academy of Sciences of the United States of America, 93, 1335-1339.

Cavanagh, P. (1992). Attention based motion perception. Science, 257, 1563-1565. 
Challinor, K. L., \& Mather, G. (2010). A motion-energy modelpredicts the direction discrimination and MAE duration of two-stroke apparent motion at high and low retinal illuminance. Vision Research, 50, 1109-1116.

Chance, F. S., Nelson, S. B., \& Abbott, L. F. (1998). Synaptic depression and the temporal response characteristics of V1 cells. Journal of Neuroscience, 18, 4785-4799.

Culham, J., Verstraten, F. A. J., Ashida, H., \& Cavanagh, P. (2000). Independent aftereffects of attention and motion. Neuron, 28, 607- 615.

Daelli, V., van Rijsbergen, N., \& Treves, A. (2010). How recent experience affects the perception of ambiguous objects. Brain Research, 1322, 81-91.

Emerson, R. C., Bergen, J. R., \& Adelson, E. H. (1992). Directionally selective complex cells and the computation of motion energy in cat visual cortex. Vision Research, 32, 203-218.

Fuortes, M. G., \& Hodgkin, A. L. (1964). Changes in time scaleand sensitivity in the ommatidia of Limulus. Journal of Physiology, 172, 239-263.

Georgeson, M. A., \& Scott-Samuel, N. E. (1999). Motion contrast: a new metric for direction discrimination. Vision Research, 39, 4393-4402.

Giaschi, D., Douglas, R., Marlin, S., \& Cynader, M. (1993). The time course of direction-selective adaptation in simple and complex cells in cat striate cortex. Journal of Neurophysiology, 70, 20242034.

Glasser, D. M., Tsui, J. M. G., Pack, C. C., \& Tadin, D. (2011). Perceptual and neural consequences of rapid motion adaptation. Proceedings of the National Academy of Sciences of the United States of America, 108(45).

Finlayson, P. G., \& Cynader, M. S. (1995). Synaptic depression in visual cortex tissue slices: An in vitro model for cortical neuron adaptation. Experimental Brain Research, 106, 145-155. 
Groppe, D. M., Urbach, T. P., \& Kutas, M. (2011). Mass univariate analysis of event-related brain potentials/fields I: A critical tutorial review. Psychophysiology, 48(12), 1711-1725.

Haug, B. A., Baudewig, J., \& Paulus, W. (1998). Selective activation of human cortical area V5A by a rotating visual stimulus in fMRI; implication of attentional mechanisms. Neuroreport, 9, 611614.

Hershenson, M. (1993). Linear and rotation motion aftereffects as a function of inspection duration. Vision Research, 33, 1913-1919.

Hempel, C. M., Hartman, K. H., Wang, X.-J., Turrigiano, G. G., \& Nelson, S. B. (2000). Multiple forms of short-term plasticity at excitatory synapses in rat medial prefrontal cortex. Journal of Neurophysiology, 83, 3031-3941.

Holm, S. (1979). A simple sequentially rejective multiple test procedure. Scandinavian Journal of Statistics, 6, 65-70.

Huk, A. C., Ress, D., \& Heeger, D. J. (2001). Neuronal basis of the motion aftereffect reconsidered. Neuron, 32, 161-172.

Kanai, R., \& Verstraten, F. A. (2005). Perceptual manifestations of fast neural plasticity: Motion priming, rapid motion aftereffect and perceptual sensitization. Vision Research, 45, 3109-3116.

Lingnau, A., Ashida, H., Wall, M. B., \& Smith, A. T. (2009). Speed encoding in human visual cortex revealed by fMRI adaptation. Journal of Vision, 9(13):3, 1-14.

Lisberger, S., \& Movshon, J. (1999). Visual motion analysis for pursuit eye movements in area MT of macaque monkeys. Journal of Neuroscience, 19, 2224-2246.

Mather, G., Pavan, A., Campana, G., \& Casco, C. (2008). The motion aftereffect reloaded. Trends in Cognitive Sciences, 12, 481-487. 
Moutoussis, K., \& Zeki, S. (1997). A direct demonstration of perceptual asynchrony in vision. Proceedings of the Royal Society of London B, 264(1380), 393-399.

Nelson, S. B. (1991). Temporal interactions in the cat visual system: I. Orientation-selective suppression in the visual cortex. Journal of Neuroscience, 11, 344-356.

Pavan, A., Campana, G., Guerreschi, M., Manassi, M., \& Casco, C. (2009). Separate motiondetecting mechanisms for first- and second-order patterns revealed by rapid forms of visual motion priming and motion aftereffect. Journal of Vision, 27, 1-16.

Pavan, A., Campana, G., Maniglia, M., \& Casco, C. (2010). The role of high-level visual areas in short- and longer-lasting forms of neural plasticity. Neuropsychologia, 48, 3069-3079.

Pavan, A., Contillo, A., \& Mather, G. (2013). Modelling adaptation to directional motion using the Adelson-Bergen energy sensor. PloS One 8(3):e59298.

Pavan, A., \& Skujevskis, M. (2013). The role of stationary and dynamic test patterns in rapid forms of motion aftereffect. Journal of Vision, 10, 1-17.

Pinkus, A., \& Pantle, A. (1997). Probing visual motion signals with a priming paradigm. Vision Research, 37, 541-552.

Priebe, N. J., Churchland, M. M., \& Lisberger, S. G. (2002). Constraints on the source of short-term motion adaptation in macaque area MT: I. The role of input and intrinsic mechanisms. Journal of Neurophysiology, 88, 354-369.

Priebe, N. J., \& Lisberger, S. G. (2002). Constraints on the source of short-term motion adaptation in macaque area MT. II. tuning of neural circuit mechanisms. Journal of Neurophysiology, 88, 370382.

Rainville, S. J., Makous W. L., \& Scott-Samuel, N. E. (2004). Opponent-motion mechanisms are self-normalizing. Vision Research, 45, 1115-1127. 
Rainville, S. J., Scott-Samuel, N. E., \& Makous, W. L. (2002). The spatial properties of opponentmotion normalization. Vision Research, 42, 1727-1738.

Rees, G., Frith, C. D., \& Lavie, N. (1997). Modulating irrelevant motion perception by varying attentional load in an unrelated task. Science, 278, 1616-1619.

Rolls, E.T., 1989. The representation and storage of information in neuronal networks in the primate cerebral cortex and hippocampus. In: Durbin, R., Miall, C., Mitchison, G. (Eds.), The Computing Neuron, Ch. 8. Addison-Wesley, Wokingham, England, pp. 125-159.

Rushton, W. A. H. (1962). Visual adaptation. Proceedings of the Royal Society B, 986, 20-46.

Seidemann, E., \& Newsome, W. T. (1999). Effect of spatial attention on the responses of area MT neurons. Journal of Neurophysiology, 81, 1783-1794.

Stratford, K. J., Tarczy-Hornuch, K., Martin, K. A. C., Bannister, N. J., \& Jack, J. J. B. (1996). Excitatory synaptic inputs to spiny stellate cells in cat visual cortex. Nature, 382, 258-261.

Strout, J. J., Pantle, A., \& Mills, S. L. (1994). An energy model of interframe interval effects in single-step apparent motion. Vision Research, 34, 3223-3240.

Takeuchi, T., \& De Valois, K. K. (1997). Motion-reversal reveals two motion mechanisms functioning in scotopic vision. Vision Research, 37, 745-755.

Taylor, M. M. (1963). Tracking the decay of the after-effect of seen rotary movement. Perceptual and Motor Skills, 16, 119-129.

Treue, S., \& Maunsell, J. H. (1996). Attentional modulation of visual motion processing in cortical areas MT and MST. Nature, 382, 539-541.

Treue, S., \& Maunsell, J. H. (1999). Effects of attention on the processing of motion in macaque middle temporal and medial superior temporal visual cortical areas. Journal of Neuroscience, 19, 7591-7602. 
van de Grind, W. A., Lankheet, M. J. M., \& Tao, R. (2003). A gain-control model relating nulling results to the duration of dynamic motion aftereffects. Vision Research, 43, 117-133.

Vautin, R. G., \& Berkley, M. A. (1977). Responses of single cells in cat visual cortex to prolonged stimulus movement: neural correlates of visual aftereffects. Journal of Neurophysiology, 40, 10511065.

Varela, J. A., Song, S., Turrigiano, G. G., \& Nelson, S. B. (1999). Differential depression at excitatory and inhibitory synapses in visual cortex. Journal of Neuroscience, 19(11), 4293-4304.

Verstraten, F. A. J., \& Ashida, H. (2005). Attention-based motion perception and motion adaptation: What does attention contribute? Vision Research, 45, 1313-1319.

Wark, B., Fairhall, A., \& Rieke, F. (2009). Timescales of inference in visual adaptation. Neuron, 61(5), 750-761.

Wexler, M., Glennerster, A., Cavanagh, P., Ito, H., \& Seno, T. (2013). Default perception of highspeed motion. Proceedings of the National Academy of Sciences of the United States of America, 110, 7080-7085.

Zengel, J. E., \& Magleby, K. L. (1982). Augmentation and facilitation of transmitter release. A quantitative description at the frog neuromuscular junction. Journal of General Physiology, 80, $583-611$. 